\title{
$\widehat{A}$ Madridge \\ madridge Journal of Dermatology \& Research \\ interconnecting Scientific World
}

Clinical Image Article

Open Access

\section{The Association of Diffuse Alopecia Areata and Psoriasis Vulgaris in a Young Child}

\author{
Younes Barbach*, Mohammed Chaouche, Abdellah Dah Cherif, Salim Gallouj and Fatima Zahra Mernissi \\ Department of Dermatology, CHU Hassan II, Fes, Morocco
}

\section{Article Info}

\section{*Corresponding author: \\ Younes Barbach}

Department of Dermatology

CHU Hassan II

Fes

Morocco

E-mail: dr.younes2011@gmail.com

Received: October 8, 2018

Accepted: October 24, 2018

Published: October 31, 2018

Citation: Barbach Y, Chaouche M, Dah Cherif A, Gallouj S, Mernissi FZ. The Association of Diffuse Alopecia Areata and Psoriasis Vulgaris in a Young Child. Madridge J Dermatol Res. 2018; 3(2): 81-82. doi: $10.18689 / \mathrm{mjdr}-1000120$

Copyright: @ 2018 The Author(s). This work is licensed under a Creative Commons Attribution 4.0 International License, which permits unrestricted use, distribution, and reproduction in any medium, provided the original work is properly cited.

Published by Madridge Publishers

\section{Clinical Image}

Psoriasis is one of the most prevalent systemic inflammatory diseases, affecting approximately $2 \%$ to $3 \%$ of the population and more than 125 million patients worldwide. Previous studies provide evidence that there is a greater frequency of autoimmune diseases among patients with psoriasis than in the general population, and many inflammatory autoimmune diseases are a result of derangements in multiple cytokine pathways [1].

The association between psoriasis and alopecia areata was still in conflict, until Szu-Ying Chu and et al. [2], conducted research in Taiwan demonstrating that patients with alopecia areata had a higher risk of developing psoriasis compared to general population. Psoriasis is classified in a category of diseases related to Th1 pathway cytokines, including interferon- $g$, IL-12 interleukin, and IL-2, which are also important in the pathogenesis of alopecia areata. Therefore, a correlation between these two diseases was conceivable [3]. We report the case of 14-year-old child, without significant pathological ATCD. Who had an occipital alopecian patch since the age of 10 , which progressively worsened by the extension of the lesion and a depilation of the eyebrows, 2 years later, the appearance of several erythematous lesions sitting at the level of the 4 limbs and then extending over the rest of the body evolving in a context of conservation of the general state. Dermatological examination revealed the presence of multiple diffuse squamous erythematous patches in the whole body (Figure 1) as well as in the vertex, infiltrated with positif methodic scratching of Brocq, a diffuse alopecic scar patch of the scalp (Figure 2).

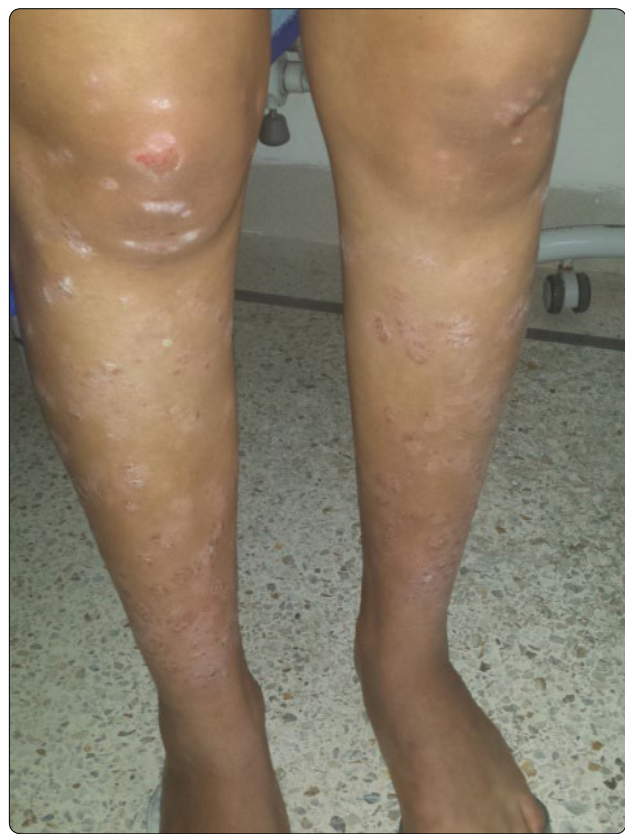

Figure 1. Multiple diffuse squamous erythematous patchs in the whole body. 


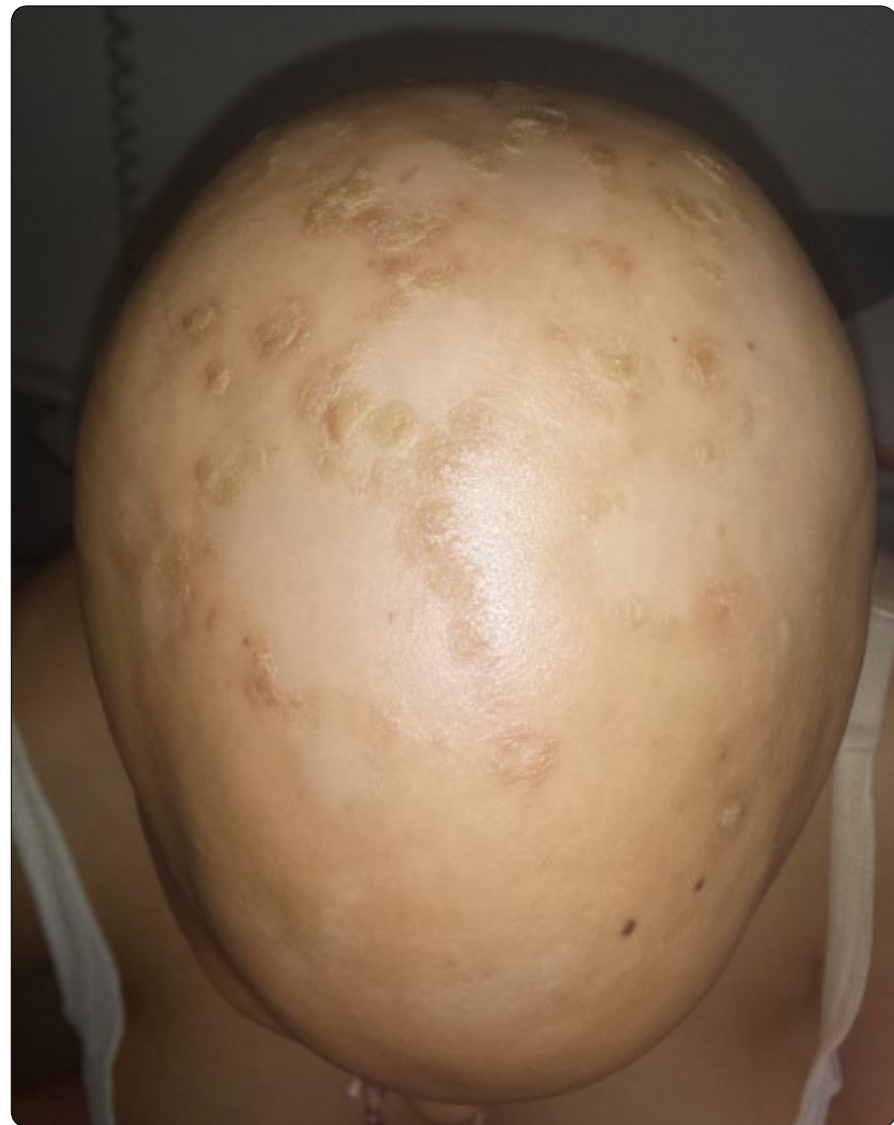

Figure 2. Diffuse alopecia areata with psoriasic patchs in the sclap.

The examination also revealed a depilation of eyebrows and eyelashes. Dermoscopy of the scalp showed cicatricial alopecia in places, yellow dots, black dots, cadaverous hair (Figure 3) and for patches, it showed a diffuse dotted vascularization corresponding to psoriasis vulagris (Figure 4).

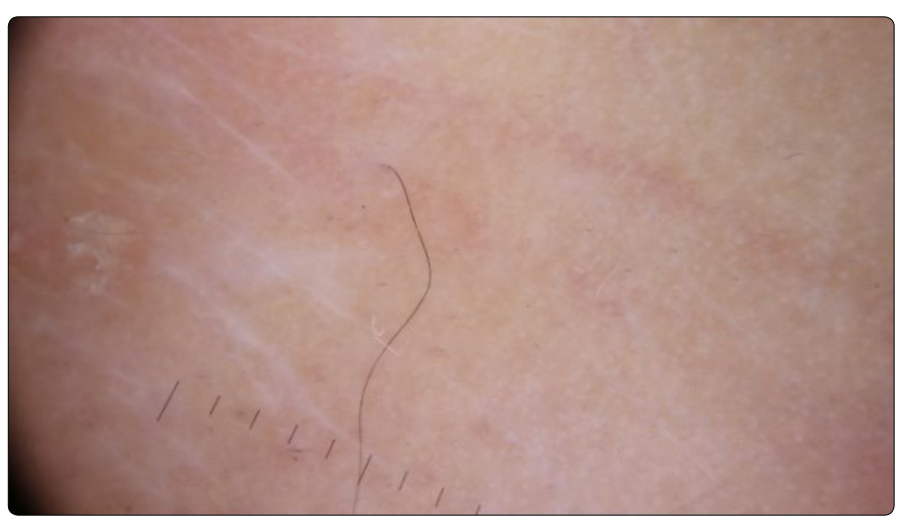

Figure 3. Dermoscopy of the scalp showed a cicatricial alopecia in places with cadaverous hair and black dots;

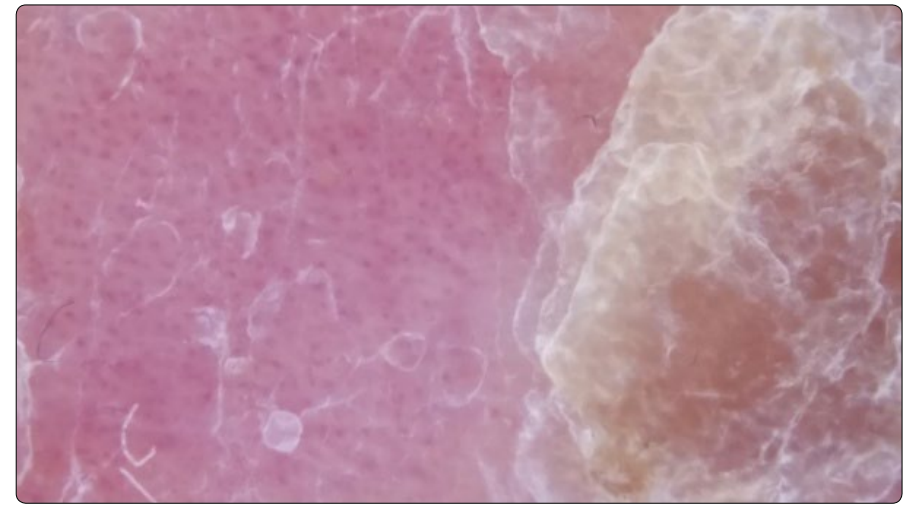

Figure 4. Dermoscopy of the patchs showed a diffuse dotted vascularization corresponding to psoriasis vulgaris.

The patient was put on methotrexate at a dose of $15 \mathrm{mg} /$ week, topical corticosteroids, minoxidil $2 \%$ for the scalp (despite the fact that minoxidil is indicated from the age of 18 according to the FDA, but from our experience and patient decline, it has given good results in children aged $\geq 8$ for various indications such as alopecia areata without any major side effects), and topical corticosteroids with cold cream preparation on the lesions of the body, with good clinical improvement after 3 months of treatment.

\section{Conflict of Interest}

The authors declare no conflicts of interest.

\section{References}

1. Wu JJ, Nguyen TU, Poon KY, Herrinton $L$. The association of psoriasis with autoimmune diseases. J Am Acad Dermatol. 2012; 67(5): 924-930. doi: 10.1016/j.jaad.2012.04.039

2. Chu SY, Chen YJ, Tseng WC, et al. Comorbidity profiles among patients with alopecia areata: the importance of onset age, a nationwide population-based study. J Am Acad Dermatol. 2011; 65(5): 949-956. doi: 10.1016/j.jaad.2010.08.032

3. Ishak RS, Piliang MP. Association between alopecia areata, psoriasis vulgaris, thyroid disease, and metabolic syndrome. J Investig Dermatol Symp Proc. 2013; 16(1): 56-57. doi: 10.1038/jidsymp.2013.22 